\title{
White thrombi in massive subchoroidal haemorrhage: indicators of the site of its origin and of the mechanism of its control
}

\author{
J REIMER WOLTER
}

From the Departments of Ophthalmology and Pathology of the University of Michigan Hospitals, Ann Arbor, Michigan, USA

SUMmARY Sharply circumscribed white thrombi with well developed Zahn's lines resembling the free-floating ball thrombi occurring in the auricles of the heart are demonstrated histologically at the end of ruptured ciliary arteries, causing massive subchoroidal haemorrhages in two degenerated human eyes. Not only do white thrombi in subchoroidal haemorrhages, in principle, serve as indicators for the exact location of the bleeding vessel, but their formation also allows for an understanding of the natural control of arterial bleeding in the eye.

Clearly demarcated white thrombi with Zahn's lines are a new and significant observation in eyes with massive subchoroidal haemorrhages. However, white thrombi are very well known to general pathologists. $^{12}$ The principles of white thrombus formation are simple: when flowing blood leaves the lumen of blood vessels covered by endothelium or makes contact with areas of endothelial damage within blood vessels, blood platelets become activated. They cause local precipitation of fibrin from the blood serum. The resulting coagulum sticks to the exposed tissues or to the area of damaged endothelium. Erythrocytes are not usually involved. This is how small blood vessels are patched until the endothelium under the coagulum can regenerate.

In larger endothelial defects or vascular rupture the coagulum of blood platelets and fibrin sticks to the margin of exposed tissues and grows by the coalescence of more platelets and fibrin, until a socalled white thrombus is formed. In the end this will be massive and stop even the fast blood flow originating from an arterial rupture. When the formation of white thrombi takes place in flowing blood, linear markings known as Zahn's lines ${ }^{1}$ typically develop. These indicate progressive growth of white thrombi without primary involvement of erythrocytes. Like sandbanks in winding rivers, Zahn's lines in white thrombi form in zones of locally slowing blood flow. ${ }^{2}$

Correspondence to J Reimer Wolter, MD, Department of Ophthalmology, University of Michigan Hospitals, Ann Arbor, Michigan 48109, USA.
Histologically these lines are seen as coralloid or concentric linear patterns.

To give a histopathological demonstration of well developed white thrombi at the distal end of ruptured ciliary arteries causing massive bleeding in the subchoroidal space is the purpose of the present paper.

\section{Case reports}

\section{CASE 1}

This 45 -year-old white male had a 10-year history of absolute glaucoma resulting from traumatic corneal perforation in his right eye. In addition to being totally blind the eye had become unsightly because of corneal scarring and neovascularisation. In January 1984 the patient developed severe pain and irritation in this eye. Examination by his local ophthalmologist revealed rupture of a staphyloma temporal to the limbus associated with a total hyphaema. Enucleation was recommended and performed by this ophthalmologist on 9 January 1984. In the process of enucleating the soft eye with its anterior rupture, another hole was made in the posterior sclera, when the optic nerve was cut in the plane of the optic nerve head. But the ocular contents remained intact and were completely removed. The eye was placed in $10 \%$ formalin for fixation and sent for pathological examination.

At gross examination the eye measured $28 \times 21 \times 20$ $\mathrm{mm}$. The cornea was diffusely scarred but was of normal size. A staphyloma in the region of the ciliary 


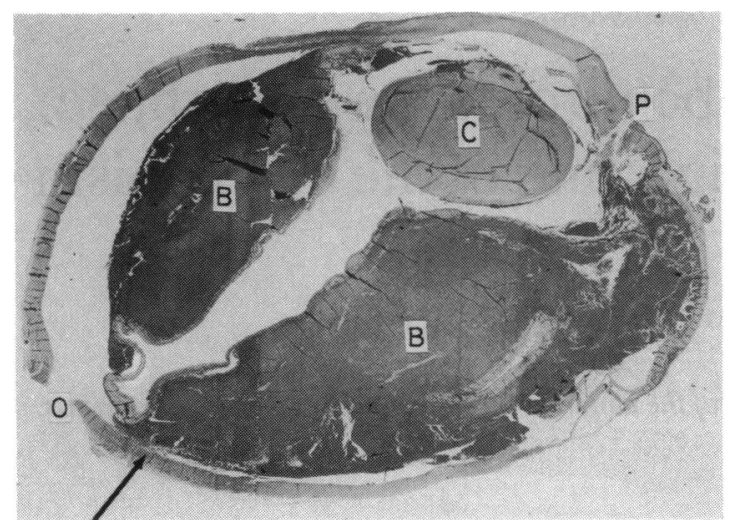

Fig. 1 Case 1. Cross-section of eye in horizontal plane showing perforated staphyloma $(\mathrm{P})$, cataract $(\mathrm{C})$, massive blood (B) in the subchoroidal space, and scleral hole in the place of the optic nerve (O). Arrow indicates area of the arterial rupture. (Paraffin section, $H$ and $E$ stain, $\times 3$ ).

body on the temporal side was ruptured. Another round hole was found in the sclera near the optic nerve, but the structures of the optic nerve head with the surrounding uvea were preserved in their continuity. The vitreous space had not been opened. No uveal tissues were exposed in the region of this posterior hole. When the eye was opened in a horizontal plane, the anterior chamber was found to be very shallow, and the lens was large and cataractous. The choroid and retina were totally detached as a unit. A massive haemorrhage filled the subchoroidal space.

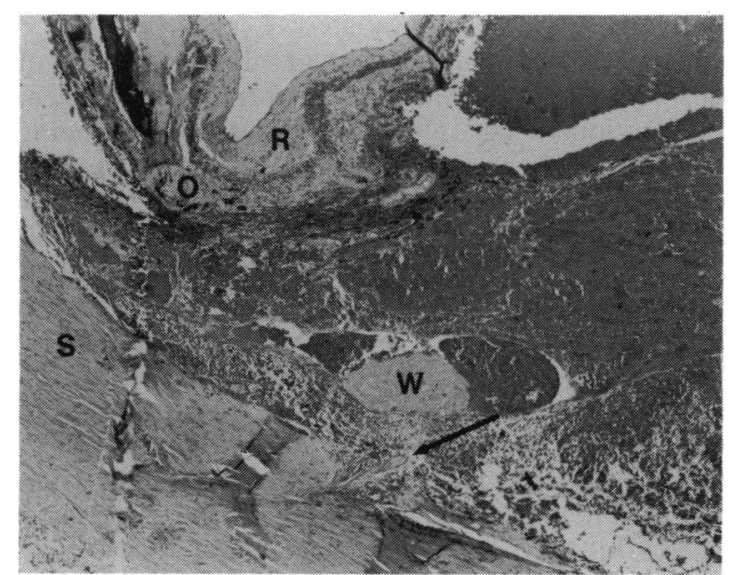

Fig. 2 Case 1. Low-power view of the white clot (W) surrounded by massive subchoroidal haemorrhage with detached choroid and retina $(\mathrm{R})$ above and sclera (S) below. Portions of optic disc $(\mathrm{O})$ are seen and arrow points to ruptured ciliary arteries. (Paraffin section, $H$ and $E$ stain, $\times 40)$.
Histological study of sections cut in the horizontal plane revealed pannus formation and general degeneration in the cornea. The filtration angle was obstructed by peripheral anterior synechiae. The lens was cataractous and showed fibrous metaplasia of anterior lens epithelium as well as degeneration and calcification of lens fibres. The clinically and grossly observed rupture of the staphyloma on the temporal aspect of the anterior sclera was histologically confirmed (Fig. 1), and there was advanced atrophy of the ciliary body in the region of the staphyloma. The retina showed mainly the type of atrophy of its innermost layers that is typical of advanced glaucoma. Retina, pigment epithelium, and choroid were detached as a unit (Fig. 1). A massive haemorrhage composed of very well preserved erythrocytes filled the subchoroidal space (Fig. 1). The sclera was thinned and perforated in the region of the staphyloma. It had an additional hole posteriorly, where the optic nerve had been cut off during the enucleation (Figs. 1 and 2). The continuity of retina and optic disc was preserved in the region of the posterior scleral hole.

The stumps of two adjacent and relatively large branches of a posterior ciliary artery, ruptured next to their exit from the scleral canal into the plane of the posterior choroid, were found on the outer aspect of the pooled subchoroidal haemorrhage-widely separated by this from the further course of these arterial branches in the detached choroid (Figs. 2-4). The sharply outlined formation of a typical white thrombus with distinctly visible lines of Zahn was seen at the inner aspect of the two arterial stumps (Figs. 2-4). This white clot was a double formation joined into one as result of the close proximity of the two

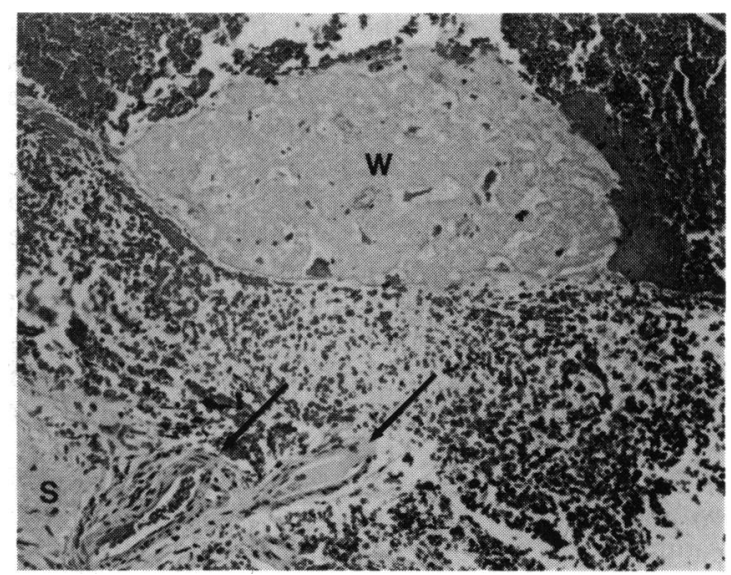

Fig. 3 Case 1. Higher power view of the white clot (W) and the two ruptured ciliary arteries (arrows) seen in Fig. 2. A portion of sclera (S) on lower left. (Paraffin section, $H$ and $E$ stain, $\times 150$ ). 


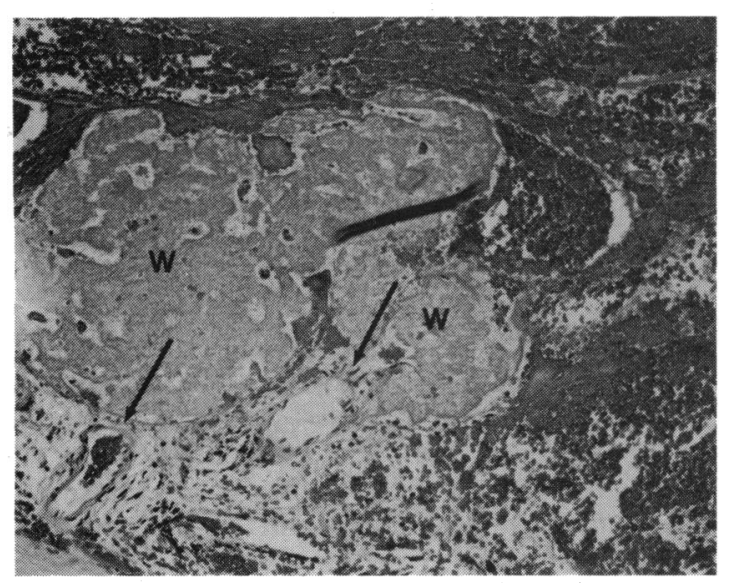

Fig. 4 Case 1. Joined double white clots (W, W) above two ruptured ciliary arteries (arrows) in the pool of subchoroidal blood. Small portion of sclera seen in left lower corner.

(Paraffin section, $H$ and $E$ stain, $\times 300$ ).

leaking arteries (Fig. 4). The white clot was surrounded by the pool of relatively fresh blood of the massive subchoroidal haemorrhage. The lumen of the arterial stumps was filled by a mixture of exudatelike plasma and some blood indicating absence of blood flow in these arteries at the time of the enucleation (Fig. 4).

CASE 2

This 34-year-old male with juvenile-onset type of diabetes mellitus had a history of proliferative diabetic retinopathy and repeated vitreous haemorrhages in his left eye. The eye was treated with photocoagulations in 1981 and 1982 . Vitrectomy and extraction of a secondary cataract were done in 1983. Retinal detachment developed later in 1983, and an operation for it was unsuccessful. The eye had become completely blind by December 1983 . Several episodes of extensive intraocular bleeding were observed. Rubeosis and blood staining of the cornea developed. The eye became hypotonic, and this was explained by detachment of the ciliary body associated with the pull of a shrinking scar of organised vitreous. Additional bleeding finally caused a rise of intraocular pressure and much pain early in 1984. The blind and painful left eye was enucleated on 23 February 1984, fixed in formalin, and sent for pathological examination.

The eyeball measured $23 \times 19 \times 19 \mathrm{~mm}$. The cornea was involved with blood staining and scarring, but it was not shrunken. The anterior chamber was deep and filled with blood. Iris and totally detached retina were involved with massive scarring in the centre of the eye. Blood filled the subretinal and subchoroidal spaces. The blood in the subchoroidal space appear-

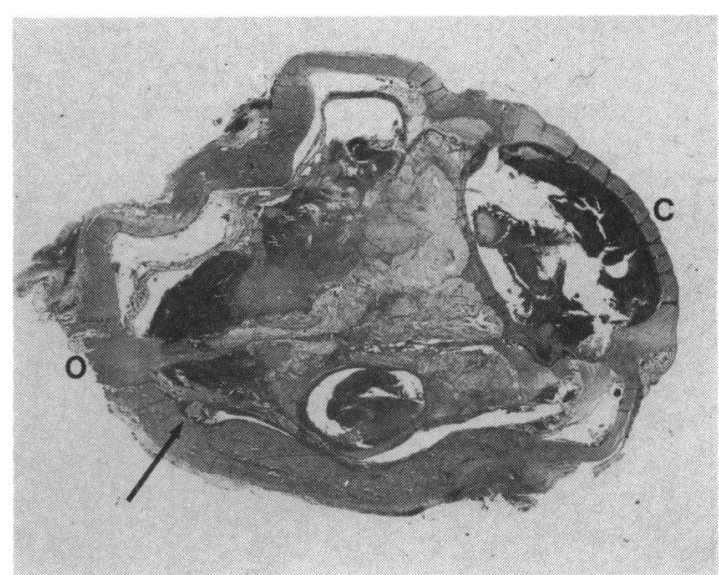

Fig. 5 Case 2. Cross-section of eye in horizontal plane with cornea (C), blood in anterior chamber, aphakia, total retinal detachment, subretinal haemorrhage in different stages, choroidal detachment, and subchoroidal haemorrhage containing a white clot (arrow). Optic nerve (O). (Paraffin section, $H$ and $E$ stain, $\times 3)$.

ed to be fresh and most of it ran out when the eye was opened. The sclera was thickened diffusely.

Paraffin sections cut in a horizontal plane confirmed the presence of neovascularisation, blood staining, and diffuse scarring in the cornea. The angle was open, and the trabecular meshwork contained macrophages filled with blood remnants. The iris was involved with rubeosis on its front surface, and its back was fixed to firm scar tissue containing lens remnants. This was tightly connected to the totally detached retina and formed a firm mass in the centre of the eye (Fig. 5). The retroretinal space was filled with a mixture of fresh and partly organised old haemorrhage. Ciliary body and choroid were also totally detached and had remnants of fresh blood in the subchoroidal space (Fig. 5). The sclera showed the diffuse thickening typical for phthisis.

The stump of a ruptured posterior ciliary artery was found surrounded by a pool of fresh subchoroidal haemorrhage near its inner scleral exit in the region of the posterior pole (Figs. 5, 6). A very well developed white clot with clear outer limits and distinct Zahn's lines had formed within this subchoroidal haemorrhage at the end of the arterial stump. The arterial lumen was histologically seen to be open towards the clot. The lumen of the artery contained exudate mixed with blood (Fig. 6). In this case another adjacent ciliary artery on its way into the choroid had remained continuous. Most choroidal arteries and retinal arterioles in this eye had advanced arteriolosclerotic changes, but the ruptured ciliary artery did not show obvious signs of severe involvement of this kind (Fig. 6). 


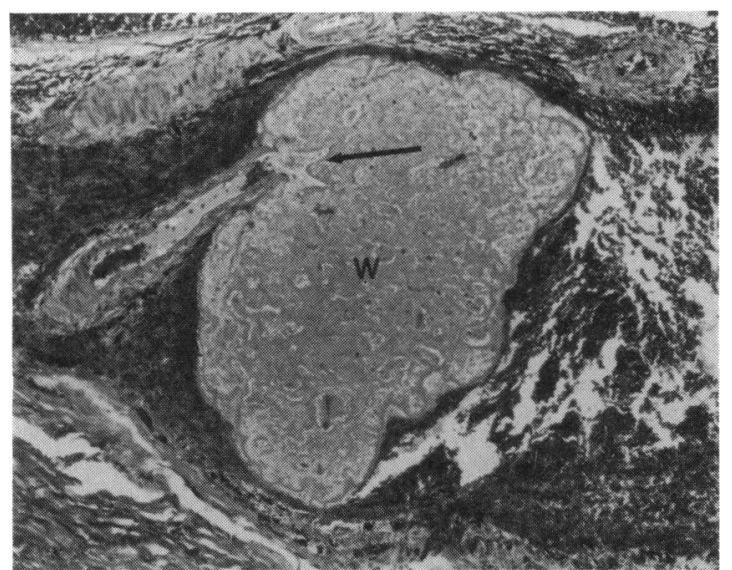

Fig. 6 Case 2. The white clot (W) of Fig. 5 seen at higher power, surrounded by subchoroidal haemorrhage above open lumen (arrow) of separated ciliary artery. A continuous ciliary artery seen above white clot. Portion of sclera below. (Paraffin section, $H$ and $E$ stain, $\times 150$ ).

\section{Discussion}

Sudden hypotony is the commonest cause of massive arterial haemorrhage into the potential subchoroidal space of the eye. This type of bleeding is known as expulsive haemorrhage ${ }^{3}$ when it occurs during surgery, but it is very similar in cases with sudden hypotony due to other causes. Intraocular arteries are not built to withstand variations in blood pressure without the support of the intraocular pressure. They have a tendency to burst when the supporting intraocular pressure is lost. In a study of the eye of a 71-year-old man with expulsive haemorrhage complicating a cataract operation rupture of the long posterior ciliary artery on the nasal side at its scleral exit was recognised as the cause of the massive subchoroidal bleeding. Granulation tissue containing new-formed primitive blood channels had formed at the distal end of the artery three weeks following the rupture in that case. ${ }^{3}$

The white thrombi at the distal end of ruptured ciliary arteries in the present cases were important to the eye pathologist for the localisation of the arterial bleeding. It is much easier to find the relatively large white thrombi in serial sections than it is to detect the ruptured arteries. The double arterial ruptures in the first case reported here indicate that massive arterial haemorrhage into the subchoroidal space can be due to more than one ruptured ciliary artery.

As elsewhere, white thrombi in massive haemorrhages of the inner eye are understood as nature's first repair of bleeding arteries. The mechanism of this repair should be well understood by the surgeon. For this mechanism to function the patient needs a good supply of blood platelets. To slow the arterial bleeding mechanically, when this has occurred during surgery, also makes sense. This is done by the time-honoured temporary tying of corneal sutures and additional application of finger pressure to the eye. Early drainage of a massive subchoroidal haemorrhage by sclerotomy, in contrast, would probably not help with the formation of a white thrombus. An early sclerotomy gives an outflow to the otherwise stagnant pool of blood in the subchoroidal space and could thus decrease the chances of the quickest possible thrombus formation. A sclerotomy can be done after the thrombus has formed and has caused the bleeding to stop. ${ }^{4-6}$

White thrombi occurring at the site of vascular rupture in the eye, in general, are not only a stage of vascular repair, for the substance of these thrombi also serves as the matrix that supports the new vascular channels which form in place of the interrupted ones. Basic knowledge about white thrombi will thus be helpful for an understanding and for clinical guidance on revascularisation as well as neovascularisation in all ocular tissues, including the retina.

Supported by the Research To Prevent Blindness, Inc., New York, NY, USA.

\section{References}

1 Ober WB, Friedrich Wilhelm Zahn 1845-1904: What's my line? Pathology annual 1978. New York: Appleton, Century, Crofts, 1978: 165-73.

2 Walter JB, Israel MS. General pathology. 2nd ed. Boston: Little, Brown, 1965: 733.

3 Wolter JR. Expulsive hemorrhage, a study of histopathological details. Albrecht von Graefes Arch Klin Ophthalmol 1982; 219: 155-8.

4 Verhoeff FH. Sceral puncture of expulsive subchoroidal hemorrhage following sclerotomy. Ophthalmic Rec 1915; 24: 55-99.

5 Vail D. Posterior sclerotomy as a form of treatment in subchoroidal expulsive hemorrhage. Am J Ophthalmol 1938; 21: 256-60.

6 Wolter JR. Expulsive hemorrhage during retinal detachment surgery: a case with survival of the eye after Verhoeff sclerotomy. Am J Ophthalmol 1961; 51: 264-6. 